\title{
Axon Regeneration in Goldfish and Rat Retinal Ganglion Cells: Differential Responsiveness to Carbohydrates and cAMP
}

\author{
Yiming Li, ${ }^{1,2 *}$ Nina Irwin, ${ }^{1,2 *}$ Yuqin Yin, ${ }^{1,2 *}$ Marc Lanser, ${ }^{4}$ and Larry I. Benowitz ${ }^{1,2,3}$ \\ ${ }^{1}$ Laboratories for Neuroscience Research in Neurosurgery, Children's Hospital, ${ }^{2 D}$ Department of Surgery, and ${ }^{3}$ Program in Neuroscience, Harvard Medical \\ School, and ${ }^{4}$ Boston Life Sciences, Inc., Boston, Massachusetts 02115
}

\begin{abstract}
Mammalian retinal ganglion cells (RGCs) do not normally regenerate their axons through an injured optic nerve, but can be stimulated to do so by activating macrophages intraocularly. In a cell culture model of this phenomenon, we found that a small molecule that is constitutively present in the vitreous, acting in concert with macrophage-derived proteins, stimulates mature rat RGCs to regenerate their axons if intracellular cAMP is elevated. In lower vertebrates, RGCs regenerate their axons spontaneously in vivo, and in culture, the most potent axon-promoting factor for these cells is a molecule that resembles the small vitreous-derived growth factor from the rat. This molecule was isolated chromatographically and was shown by mass spectrometry to be a carbohydrate. In agreement with this finding, D-mannose proved to be a potent axon-promoting factor for rat RGCs $\left(\mathrm{ED}_{50} \sim 10 \mu \mathrm{M}\right)$; this response was cAMP-dependent and was augmented further by macrophage-derived proteins. Goldfish RGCs showed far less selectivity, responding strongly to either D-mannose or D-glucose in a cAMP-independent manner. These findings accord well with the success or failure of optic nerves to regenerate in higher and lower vertebrates in vivo. The axon-promoting effects of mannose are highly specific and are unrelated to energy metabolism or glycoprotein synthesis.
\end{abstract}

Key words: retina; optic nerve; axon; regeneration; retinal ganglion cells; mannose; growth factors; macrophages; cAMP

\section{Introduction}

Like other CNS pathways in mature mammals, the optic nerve does not normally regenerate after injury. Retinal ganglion cells (RGCs) initiate a sprouting reaction at their damaged nerve endings, but this growth is abortive, and the cells soon begin to die (Ramon y Cajal, 1991). Nonetheless, RGCs can regenerate lengthy axons through a peripheral nerve graft (Aguayo et al., 1991) and even through the optic nerve itself if the lens is injured (Fischer et al., 2000; Leon et al., 2000) or if a fragment of peripheral nerve is implanted into the vitreous (Berry et al., 1996). These latter manipulations lead to the appearance of activated macrophages in the eye, and we have recently shown that intravitreal macrophage activation is sufficient to allow RGCs to regenerate their axons through the optic nerve (Leon et al., 2000; Yin et al., 2003). In culture, a macrophage-derived protein, acting in concert with a small molecule that is constitutively present in the

Received March 4, 2003; revised July 7, 2003; accepted July 8, 2003.

This work was supported by National Institutes of Health Grant NEI EY05690 and Boston Life Sciences, Inc.. We are grateful to Beverly Chamberlin and Douglas Gage of the Michigan State University Mass Spectrometry Facility for the MS analyses; Lijie Wang, Timo Roser, Katie Black, Miao-fen Gu, Eunice Wang, Raymond Tabibiazar, and Yun Jing for assistance at various stages of this project; Drs. F. Rolland (University of Leuven, Belgium), Phillips Robbins (Boston University School of Medicine), and Paul Rosenberg (Children's Hospital, Harvard Medical School) for helpful discussions; and David Goldberg for assistance with graphics.

*Y.L., N.I., and Y.Y. contributed equally to this work.

Correspondence should be addressed to Dr. Larry Benowitz, Children's Hospital, 300 Longwood Avenue, Boston, MA 02115. E-mail: larry.benowitz@tch.harvard.edu.

Copyright $\odot 2003$ Society for Neuroscience $\quad$ 0270-6474/03/237830-09\$15.00/0 vitreous, stimulates mature rat RGCs to regenerate their axons in a cAMP-dependent manner (Yin et al., 2003).

In contrast to mammals, fish and amphibia can regenerate their optic nerves throughout life (Jacobson, 1991). In culture, the most potent axon-promoting factor for goldfish RGCs is a small hydrophilic molecule $(<500 \mathrm{Da})$ that is secreted by nonneuronal cells of the optic nerve. We referred to this molecule as AF-1 (Schwalb et al., 1995, 1996).

We show here that the small, endogenous axon-promoting factors for goldfish and rat RGCs are carbohydrates. Rat RGCs extend axons in response to low micromolar concentrations of mannose when intracellular cAMP is elevated, and macrophagederived proteins augment outgrowth further. Goldfish RGCs show considerably less selectivity, extending axons in response to either mannose or glucose even without altering intracellular cAMP levels. These results may help shed light on the regenerative success or failure seen in the primary visual pathways of mammals and lower vertebrates in vivo.

\section{Materials and Methods}

Extraction of a low molecular weight growth factor from vitreous humor. Molecules present in the vitreous bodies of either normal adult male Fisher rats (200-250 gm; Charles River Laboratories, Wilmington, MA) or from similar rats, $7 \mathrm{~d}$ after activating macrophages in the eye, were extracted into normal saline ( 8 vitreous bodies in $1.5 \mathrm{ml}$ saline, $4^{\circ} \mathrm{C}$, with overnight mixing). Macrophages were activated by lens injury, which induces axon regeneration in vivo (Leon et al., 2000). Cellular debris was removed using a $45 \mu \mathrm{m}$ low protein-binding filter (Corning Costar, 
Cambridge, MA), and low molecular weight components were separated by ultrafiltration through a $3 \mathrm{kDa}$ molecular weight cutoff (MWCO) device (Centricon YM3; Millipore, Bedford, MA). Molecules present in the vitreous bodies from newborn calves (Research 37, Hopkinton, MA) were similarly extracted into saline ( 3 volumes, $1 \mathrm{hr}$ ) after cutting the vitreous into small pieces $\left(<125 \mathrm{~mm}^{3}\right)$, and components $<3 \mathrm{kDa}$ were separated as above. Bioactivity was tested using goldfish retinal cultures (see below) in the presence or absence of 6-thioguanine (6-TG) (20 $\mu \mathrm{M}$; Sigma-Aldrich, St. Louis, MO), an antagonist of a purine-sensitive kinase (Volonte et al., 1989), or 4-nitrobenzyl-6-thioinosine (NBTI) (20 $\mu \mathrm{M}$; Sigma-Aldrich), an inhibitor of purine transport across the cell membrane (Tolkovsky and Suidan, 1987).

Analysis of bovine vitreous conditioned media by reversed-phase HPLC. The low molecular weight extract of bovine vitreous was lyophilized and extracted into either $15 \%$ of its original volume of $95 \%$ ethanol and then water, or simply into $3 \%$ of its original volume of water. Either procedure removed $>95 \%$ of the inorganic salts while leaving essentially all of the axon-promoting activity in the soluble phase (data not shown). We applied $100 \mu \mathrm{l}$ of extract to a C18 reversed-phase HPLC column (Delta Pak $5 \mu \mathrm{m} \mathrm{C18} 100 \AA ; 0.5 \mathrm{ml} / \mathrm{min}$; Waters, Milford, MA) pre-equilibrated with mobile phase A $[0.1 \%$ trifluoroacetic acid (TFA) in water $]$ at 0.5 $\mathrm{ml} / \mathrm{min}$. The column was washed with this same buffer for $2 \mathrm{~min}$ and then eluted with $21 \mathrm{ml}$ of a $0-100 \%$ gradient of mobile phase B (isopropanol, acetonitrile, and water in a ratio of $3: 2: 2$, with $0.08 \%$ TFA) at a flow rate of $0.5 \mathrm{ml} / \mathrm{min}$. Fractions of $1 \mathrm{ml}$ were bioassayed.

Purification of the biologically active component. After extraction and desalting, the low molecular weight extract from the bovine vitreous was applied to a Sephadex G-10 column $(1.6 \times 48 \mathrm{~cm}$; separation range, $50-700 \mathrm{Da}$ ) and eluted with water at a flow rate of $0.3 \mathrm{ml} / \mathrm{min}$. Fractions of $4.5 \mathrm{ml}$ were collected and bioassayed (see below) at $\sim 5 \%$ of their original concentration in the starting sample. Fractions containing axonpromoting activity were pooled and re-chromatographed on the same column.

Final purification was achieved using normal-phase chromatography. Biologically active fractions from the G-10 column were lyophilized, redissolved in $200 \mu \mathrm{l} 75 \%$ acetonitrile (ACN), and applied at $1 \mathrm{ml} / \mathrm{min}$ to a pre-equilibrated LC- $\mathrm{NH}_{2}$ HPLC column (Supelco aminopropyl Supelcosil LC- $\mathrm{NH}_{2}$; Sigma-Aldrich). The column was eluted with $75 \% \mathrm{ACN}$, collecting $1 \mathrm{ml}$ fractions, which were bioassayed. Fractions containing axon-promoting activity were pooled, re-chromatographed on the same column, and active fractions were again identified by bioassay.

Goldfish retinal ganglion cell cultures. Retinas from anesthetized, darkadapted, Comet variety goldfish (Mt. Parnell Fisheries, Ft. Loudon, PA) were dissected, digested with papain, and triturated to yield an RGCenriched cell suspension (Schwartz and Agranoff, 1981; Schwalb et al., 1995). Approximately 500 RGCs were plated into each well of a 24 -well plate (Nunc, Rochester, NY) in Liebovitz' L15 media (Invitrogen, Carlsbad, CA) supplemented with insulin, antioxidants, and other components as described elsewhere (Schwalb et al., 1995). Experimental samples, along with positive and negative controls, were tested in quadruplicate in randomized positions on the plate. After $6 \mathrm{~d}$ in culture at $25^{\circ} \mathrm{C}$, the fluorescent vital dye Calcein (final concentration, $5 \mu \mathrm{M}$; Molecular Probes, Eugene, OR) was added, and axon outgrowth, defined as the percentage of RGCs that extended an axon $\geq 5$ cell diameters in length, was evaluated in 150 consecutively encountered RGCs per well by an observer blind to treatment conditions. Data were analyzed by averaging axon growth across the four wells for each sample, subtracting the level of growth in negative controls (typically $2-5 \%$ ), and normalizing by the net growth in positive controls (pre-validated AF-1 unless stated otherwise: growth usually $20-40 \%$ ). Data are presented as normalized means \pm SEM. Normalization was done to be able to compare results across experiments, which sometimes differed considerably in their baseline and peak growth levels. Major findings were replicated in multiple independent experiments. Unless noted otherwise, all molecules were analytical grade from Sigma-Aldrich. Mannoheptulose $(\mathrm{MH})$ was from CMS Chemicals, Ltd. (Abington, UK), and forskolin was from Alamone Labs (Jerusalem, Israel).

Rat retinal ganglion cell cultures. To distinguish ganglion cells from other retinal cell types, adult male Fisher rats (200-250 gm; Charles River
Laboratories) were anesthetized (ketamine, $60-80 \mathrm{mg} / \mathrm{kg}$, i.p., xylazine, $10-15 \mathrm{mg} / \mathrm{kg}$, i.p.), the superior colliculi were surgically exposed, and Fluorogold (FG) (2\% in saline, $5 \mu \mathrm{l}$; Fluorochrome, Denver, CO) was injected into several sites bilaterally. A small piece of Surgifoam (Ethicon, Somerville, NJ) impregnated with FG was placed over the superior colliculi, and the scalp wound and overlying skin were closed. After allowing $7 \mathrm{~d}$ for FG to be retrogradely transported to RGC somata, rats were killed with an overdose of anesthesia, eyes were removed, and the retinas were dissected and dissociated as described (Yin et al., 2003). Mixed cultures containing 100-150 FG-labeled RGCs per well were maintained for $3 \mathrm{~d}$ at $37^{\circ} \mathrm{C}$ in 4 -well plates using the same medium used for goldfish RGC cultures, but supplemented with $2.2 \mathrm{mg} / \mathrm{ml} \mathrm{NaHCO}$ and $5 \% \mathrm{CO}_{2}$. Experimental samples were tested in quadruplicate and were distributed across the culture dishes in a randomized manner. Statistical handling of the data were as described above, except that results are represented as fold-increase relative to the baseline growth in negative controls (generally $5-8 \%)$.

GAP-43 immunostaining. Cells cultured on coverslips were fixed with $4 \%$ paraformaldehyde $[10 \mathrm{~min}$ at room temperature (RT)], treated with $0.1 \%$ Triton X-100 and 5\% goat serum (30 min), and incubated with an anti-GAP-43 antibody (1:500 dilution, clone 9-1E12; Chemicon, Temecula, CA) $4^{\circ} \mathrm{C}$ overnight, followed by a fluorescein-conjugated goat anti-mouse secondary antibody (1:500, AlexaFluor 488, $1 \mathrm{hr}$, RT; Molecular Probes). Controls were stained omitting the primary antibody.

Macrophage-conditioned media. Proteins secreted by normal alveolar macrophages (NR8383; ATCC, Manassas, VA) were collected into serum-free $\mathrm{F}-12 \mathrm{~K}$ medium for $8 \mathrm{hr}$ at $37^{\circ} \mathrm{C}$ in $5 \% \mathrm{CO}_{2}$ and concentrated using a $3 \mathrm{kDa}$ MWCO ultrafiltration membrane as described (Yin et al., 2003).

Mass spectrometry. Fast Atom Bombardment (FAB) mass spectra were obtained at the Michigan State University Mass Spectrometry Facility using a JEOL (Peabody, MA) HX-110 double-focusing mass spectrometer operating in either the positive or negative ion mode. Ions were produced by bombardment with a beam of Xe atoms $(6 \mathrm{keV})$. The accelerating voltage was $10 \mathrm{kV}$, and the resolution was set at 3000 . For FABCAD-MS/MS, helium was used as the collision gas in a cell located in the first field-free region. The helium pressure was adjusted to reduce the abundance of the parent ion by $50 \%$. A data system generated linked scans at a constant ratio of magnetic to electrical fields (B/E). The instrument was scanned from mass/charge ratios (m/z) 50-2000. Spectra are presented from single scans.

\section{Results}

\section{The mammalian vitreous contains an axon-promoting factor similar to goldfish AF-1}

The vitreous body of the rat contains a small molecule that enables mature RGCs to regenerate their axons when intracellular cAMP is elevated (Yin et al., 2003). To determine whether this molecule is similar to AF-1, the small axon-promoting factor described in goldfish (Schwalb et al., 1995, 1996), we extracted molecules present in the rat's vitreous body into saline and separated components $<3 \mathrm{kDa}$ by ultrafiltration. When tested in goldfish retinal cultures, the low molecular weight vitreous extract $(\mathrm{VE}<3)$ induced regenerative outgrowth even when diluted 80 -fold; axon-promoting activity was high both in the normal eye and after lens injury (Fig. $1 a-c$ ). These effects were unrelated to changes in cell survival (Fig. 1d). Like the rat vitreous, bovine vitreous contains high levels of the axon-promoting activity (Fig. $1 e$ ), providing a plentiful source for purification.

Another small molecule that can stimulate goldfish RGCs to regenerate their axons is the purine nucleoside inosine (Benowitz et al., 1998). To determine whether $\mathrm{VE}<3$ resembles goldfish AF- 1 or inosine in its activity, we investigated whether its activity could be inhibited by 6-thioguanine, a purine analog that blocks the effect of AF-1 noncompetitively but acts competitively with inosine (Petrausch et al., 2000), or with NBTI, an inhibitor of purine transport across the cell membrane that blocks the effects 

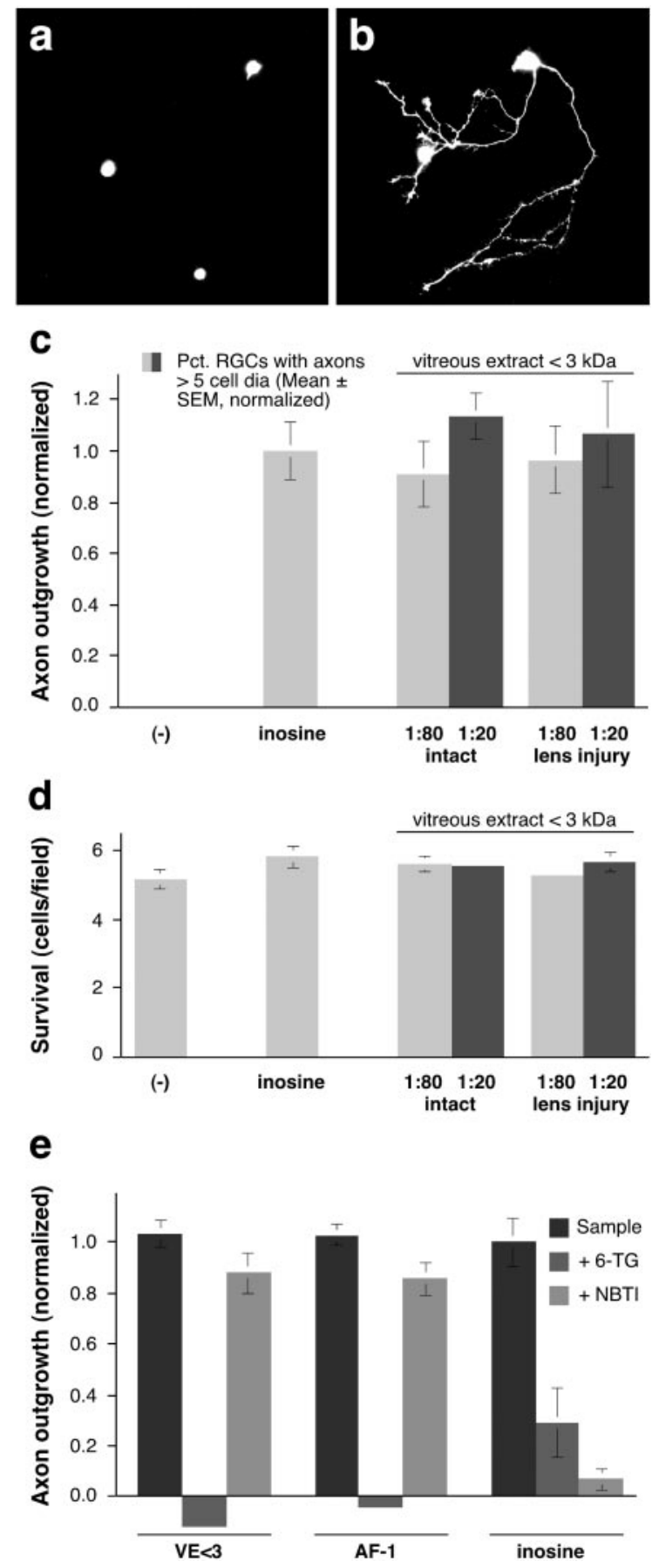

Figure 1. A low molecular weight factor extracted from the rat vitreous (VE $<3$ ) resembles goldfish AF-1. $a, b$, Dissociated goldfish RGCs cultured in defined media in the absence $(a)$ or presence $(b)$ of a low molecular weight factor extracted from the rat vitreous. Viable cells are visualized with Calcein (Molecular Probes). c, Molecules $<3 \mathrm{kDa}$, extracted from either the normal rat vitreous or from the vitreous 1 week after lens injury, induce high levels of axon outgrowth even when diluted 80 -fold. $d$, Cell survival. The number of retinal ganglion cells per $200 \times$ microscope field is unaffected by any of the factors tested. $e$, Axon growth induced by either VE $<3$ or goldfish-derived AF-1 is blocked by the purine analog 6-TG, but is unaffected by NBTI, an inhibitor of purine transport. Conversely, the effect of inosine is blocked by NBTI but is only partially inhibited by 6-TG.

of inosine but not of AF-1 (Benowitz et al., 1998). 6-TG eliminated growth induced by $\mathrm{VE}<3$ and AF-1, while only partially decreasing the effect of inosine (Fig. 1e). Conversely, NBTI had little effect on growth induced by $\mathrm{VE}<3$ or $\mathrm{AF}-1$, but blocked the effects of inosine. Thus, pharmacologically, VE $<3$ resembles goldfish AF-1.

\section{The small vitreous-derived factor stimulates adult rat RGCs to regenerate axons}

Mature rat RGCs, identified by retrograde labeling with Fluorogold, were grown in mixed, dissociated cultures in defined, serum-free media. Under these conditions, 5-8\% of RGCs extended axons $>2$ cell diameters in length by $3 \mathrm{~d}$. In isolation, VE $<3$ enhanced growth only slightly above this baseline. Neither forskolin $(15 \mu \mathrm{M})$ nor the nonhydrolyzable cAMP analog, SpcAMPs $(100 \mu \mathrm{M})$, had any effect. In the presence of either forskolin or Sp-cAMPs, however, VE $<3$ increased outgrowth approximately threefold (Fig. $2 a-e)(p<0.001)$. These effects were unrelated to changes in cell survival (Fig. 2f). Maximal effects were attained even when $\mathrm{VE}<3$ was diluted to $4 \%$ of its original concentration in the vitreous (Fig. $2 g$ ).

\section{Isolation of the active molecule}

When VE $<3$ was applied to a reversed-phase C18 HPLC column, axon-promoting activity eluted in the flow-through (Fig. $3 a, b)$. A similar elution profile was reported previously for goldfish AF-1 (Schwalb et al., 1995). Based on this hydrophilic behavior, we used normal-phase chromatography as a final step in purification.

On a gel-filtration column (Sephadex G-10), the active component of $\mathrm{VE}<3$ eluted as a single peak (165-180 min) that partially overlapped with a major $214 \mathrm{~nm}$ absorbance region (Fig. $3 c, d)$. Even at high concentrations, the active fraction induced somewhat lower outgrowth than the starting material. A component that eluted later from the G-10 column and that had no activity by itself brought the level of outgrowth induced by the active fraction back to that of the starting material (see below). Applying the active fraction from the G-10 column to a normalphase $\mathrm{LC}-\mathrm{NH}_{2}$ column resulted in a high degree of purification, with the axon-promoting factor eluting later than most other 214 $\mathrm{nm}$ absorbing components (Fig. $3 e, f$ ). The active fraction was further purified by being run again on the same $\mathrm{LC}-\mathrm{NH}_{2}$ column (data not shown).

\section{Identification of the active factor by mass spectrometry}

The active fraction from the $\mathrm{LC}-\mathrm{NH}_{2}$ column, along with adjacent inactive fractions, were analyzed by $\mathrm{FAB}$ mass spectrometry in the negative (Fig. $4 a, b$ ) or positive (Fig. $4 c, d$ ) ion modes in the presence of glycerol $(+g l y)$. In the negative ion mode, the active fraction (17) contained an ion with $\mathrm{m} / \mathrm{z}=179.2$ (Fig. 4b, arrow) that was absent in the adjacent inactive fraction (Fig. $4 a$, Fract. $16)$. Because $\mathrm{m} / \mathrm{z}$ values in the negative ion mode correspond to the true mass minus 1 proton, the molecule present in the active fraction is predicted to have a mass of 180 . In the positive ion mode, two peaks appeared in the biologically active fraction that were absent in the adjacent inactive fraction $(\mathrm{m} / \mathrm{z}=273.2$ and 255.2 ) (Fig. $4 c, d$, arrows). Because $\mathrm{m} / \mathrm{z}$ values in the positive ion mode contain an additional proton, the two unique ions are predicted to have masses of 272.2 and 254.2; however, if these ions represent glycerol adducts (mass, 92) of the parent species, the mass of the larger one would be 180 , which is similar to that found in the negative ion mode, whereas the other would be 162. Further analysis of the $\mathrm{m} / \mathrm{z}=273$ ion by MS/MS in the positive ion mode (Fig. $4 e$, curved arrow) confirmed the presence of glycerol $(\mathrm{m} / \mathrm{z}=93$, asterisk $)$ and the 180 mass $(\mathrm{m} / \mathrm{z} \sim 181$, double arrows). MS/MS also generated peaks corresponding to the parent species minus multiples of 18, i.e., 163, 145, and 127 (arrows). 

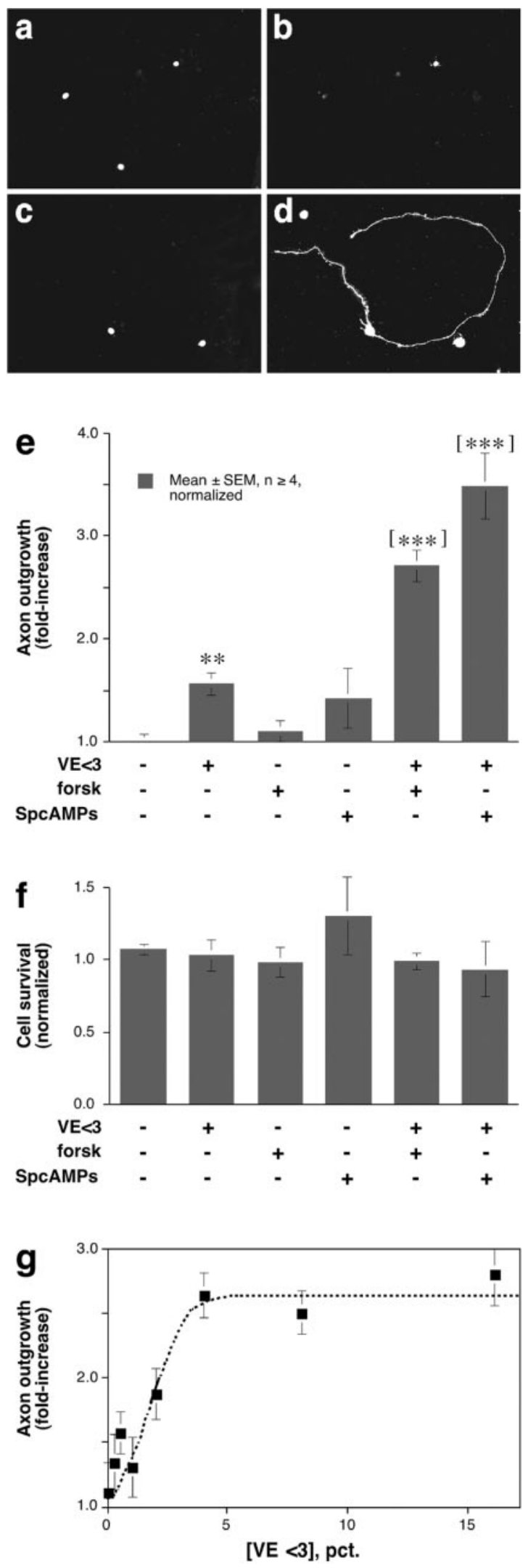

Figure 2. The small, vitreous-derived factor stimulates rat RGCs to regenerate their axons. $a-d$, Dissociated cultures of the mature rat retina were exposed to either defined media containing $15 \mu \mathrm{m}$ forskolin $(a, b)$ or the same conditions plus VE $<3$.c, d, RGCs are distinguished by retrograde FG-labeling. $a$, $c$, Axon outgrowth visualized by staining with antibodies to GAP-43 $(b, d)$.e, Quantitative results. The effects of $\mathrm{VE}<3$ are potentiated by either forskolin (forsk, 15 $\mu \mathrm{M})$ or Sp-cAMPs $(100 \mu \mathrm{M})$. Results for all rat RGC experiments are normalized to the level of
The latter peaks are likely to represent serial losses of hydroxyl groups from the 181 ion, whereas the peaks with $\mathrm{m} / \mathrm{z} \sim 255$ and 237 (arrowheads) probably represent glycerol adducts of the 163 and 145 ions, respectively. These results predict that the active molecule is a carbohydrate with the formula $\mathrm{C}_{6} \mathrm{H}_{12} \mathrm{O}_{6}$ (mass, 180).

\section{Axon regeneration in goldfish RGCs}

Based on the mass spectrometry results, we tested whether monosaccharides or related structures stimulate goldfish RGCs to regenerate their axons. The culture medium used in these studies already contains high concentrations of galactose $(5 \mathrm{~mm})$ and pyruvate $(5 \mathrm{~mm})$ as carbon sources and for energy metabolism. Thus, any effects of carbohydrates on axon outgrowth are likely to be highly specific and independent of energy metabolism. Myo-inositol, the ketoses fructose and sorbose, and the aldoses D-allose, D-altrose, D-gulose, D-talose, and D-galactose all failed to stimulate outgrowth (tested at 50-100 $\mu \mathrm{M}$ ). However, two of the carbohydrates that are present in the vitreous, mannose and glucose (Walker and Patrick, 1967), had strong axon-promoting effects (Fig. $5 a$, compare Fig. $3 d, f$ ); their L-enantiomers were inactive (Fig. 5a). These effects were independent of cell survival (Fig. 5b). Maximal effects of mannose (Fig. 5c) or glucose (data not shown) were achieved at concentrations of 25-50 $\mu \mathrm{M}$, with an $\mathrm{ED}_{50}$ of 5-10 $\mu \mathrm{M}$. Like the growth-promoting factor isolated from bovine vitreous, the activity of mannose or glucose on goldfish RGCs was augmented by the addition of a late-eluting fraction from the size-exclusion column, which itself did not cause growth (Fig. 5d). The effect of glucose (Fig. $5 e$ ) or mannose (data not shown) on goldfish RGCs was not enhanced with the membrane-permeable, non-hydrolyzable cAMP analog dibutyryl cAMP (dBcAMP). Conversely, the protein kinase A inhibitor KT5720, at the normally effective dosage of $1 \mu \mathrm{M}$, did not diminish the effect of mannose, and had only a slight effect at 10 $\mu \mathrm{M}$ (Fig. $5 f$ ). Likewise, the protein kinase A (PKA) inhibitor RpcAMPs had no effect on glucose-induced outgrowth at the normally effective dose of $100 \mu \mathrm{M}$, but blocked growth by $48 \%$ when tested at $1 \mathrm{~mm}$ (data not shown).

To further investigate structure-function relationships and possible mechanisms of action, we examined the activity of several additional compounds (Fig. 6a). D-glucosamine induced strong outgrowth $(p<0.001)$, whereas D-mannosamine did not, nor did 2-deoxy-D-glucose (2-DG), 3-O-methyl-D-glucose (3OMG), methyl- $\alpha$-D-glucopyranoside, methyl- $\beta$-D-glucopyranoside, $N$-acetylglucosamine, or fucose.

In several cell types, glucose is sensed by the activity of hexokinases, the first enzymes in the glycolytic pathway, or by the concentration of downstream metabolites (Rolland et al., 2001). In goldfish RGCs, $\mathrm{MH}$ (10 mM), an inhibitor of both glucose- 6 kinase and hexose- 6 kinase, had no effect on outgrowth induced by glucose or mannose, despite being detrimental to cell survival (Fig. 6b). Thus, the glucose-mannose sensor for axon growth is not the kinase involved in the first step of glycolysis, nor does it depend on the intracellular concentration of the 6-phosphate derivatives or of any downstream metabolites. The failure of $\mathrm{MH}$ to block outgrowth, even in the face of diminished cell survival, provides further evidence that the axon-promoting effects of D-glucose or D-mannose are unrelated to cell survival.

growth in defined media alone. $f$, None of the agents tested altered RGC survival. $g$, VE $<3$ gives a near-maximal response at a concentration of $5 \% .{ }^{* *} p<0.01$ compared with negative control; ${ }^{[* *]} p<0.001$ compared with either negative controls or cells treated with PKA agonists alone. 
When introduced extracellularly, Dglucose-6-phosphate and D-mannose-6phosphate stimulated a modest amount of outgrowth at $100 \mu \mathrm{M}(p<0.01)$ and appreciable growth at $1 \mathrm{~mm}(p<0.001)$ (Fig. $6 c)$. The 6-phosphate derivatives are negatively charged and do not pass through the cell membrane (Abeles et al., 1992). This suggests that the 6-phosphate derivatives, and by extension glucose and mannose themselves, may stimulate axon outgrowth via an extracellular sensor.

\section{Rat RGCs respond selectively to mannose}

RGCs from the mature rat showed a far greater response selectivity than goldfish RGCs. D-mannose by itself had little effect on rat RGCs (Fig. 7a), but in the presence of forskolin, it increased axon outgrowth threefold over baseline $(p<0.001)$ (Fig. $7 a)$. This effect was similar to that of VE $<3$ (compare Fig. 2), and was likewise unrelated to changes in cell survival (Fig. $7 b$ ). In the presence of forskolin, mannose elicited maximal effects by $50 \mu \mathrm{M}$, with an $\mathrm{ED}_{50}$ of $\sim 10 \mu \mathrm{M}$ (data not shown). Stereospecificity is demonstrated by the inactivity of L-mannose (Fig. 7a). Under similar conditions, glucose had no effect whatsoever (Fig. $7 a$ ), even when present at millimolar concentrations (data not shown). Of the other sugars tested, gulose had some activity $(p<$ 0.05), although well below that of mannose. Unlike goldfish RGCs, rat RGCs did not respond to glucosamine (Fig. $7 a$ ) or to high concentrations of mannose-6-phosphate (up to $10 \mathrm{~mm}$ : data not shown).

\section{Additive effect of a macrophage-derived factor or factors and mannose in rat RGCs}

In culture, a $10-20 \mathrm{kDa}$ macrophage-derived protein potentiates the response of rat RGCs to a small vitreous-derived growth factor when $[\mathrm{cAMP}]_{i}$ is elevated (Yin et al., 2003). In the presence of forskolin, macrophage-conditioned media nearly doubled the response of rat RGCs to mannose, elevating growth almost sixfold above baseline (Fig. 7c). This growth corresponds to $>50 \%$ of cultured RGCs extending axons in $3 \mathrm{~d}$.

\section{Discussion}

The present results show that the small, axon-promoting molecules present in the visual systems of goldfish and rats are simple carbohydrates. Goldfish RGCs require low micromolar concentrations of glucose or mannose to regenerate their axons, even when other energy sources are abundant. Mature rat RGCs are even more selective, responding to mannose but not glucose, and only when intracellular cAMP levels are elevated; macrophagederived proteins enhance these effects further. These findings may in part explain the markedly different capacities of lower vertebrates and higher vertebrates to regenerate their optic nerves in vivo. That is, whereas the normal abundance of glucose may suffice to enable goldfish RGCs to regenerate injured axons, rat RGCs can only respond to mannose, and only do so on a conditional basis.

\section{Selectivity in carbohydrate responsiveness}

The low molecular weight growth factor present in the mammalian vitreous was found here to be similar to the goldfish axon-
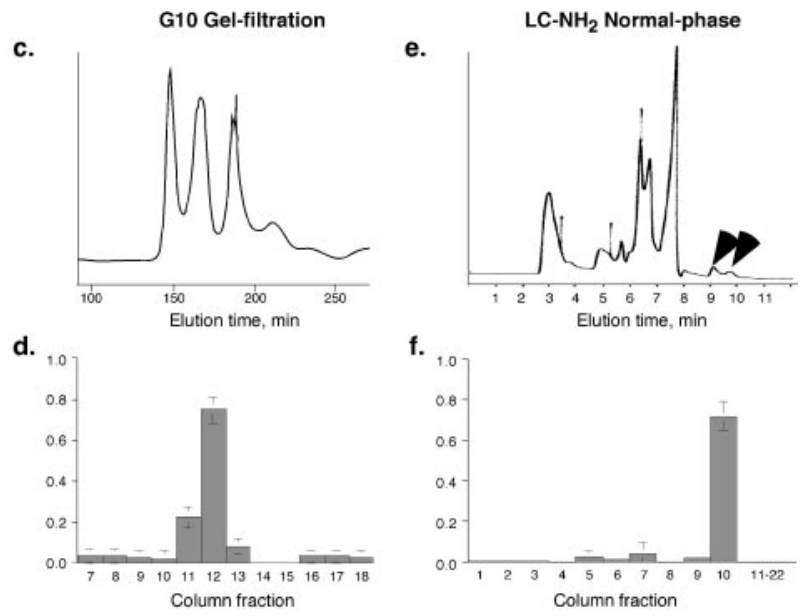

Figure 3. Isolation of the low molecular weight growth factor from bovine vitreous. $a, b$, Reversed-phase HPLC. The low molecular weight factor from bovine vitreous was concentrated, extracted into 95\% ethanol, and subjected to HPLC on a C-18 versed-phase column. Axon-promoting activity eluted early. c, d, On a G-10 Sephadex gel-filtration column, the axon列 activity from the gel-filtration column were pooled and separated on an LC- $\mathrm{NH}_{2}$ normal-phase HPLC column. Bioassays, performed on goldfish RGCs, show that the axon-promoting activity elutes later than most other components (arrows).

promoting factor, AF-1, in its bioactivity, size, hydrophilic behavior, sensitivity to 6-thioguanine, and insensitivity to NBTI (Schwalb et al., 1995, 1996; Benowitz et al., 1998; Petrausch et al., 2000). After isolation by ultrafiltration, differential solubility, gel-filtration chromatography, and normal-phase HPLC, this axon-promoting factor was found by mass spectrometry to be a carbohydrate with the formula $\mathrm{C}_{6} \mathrm{H}_{12} \mathrm{O}_{6}$. Testing the carbohydrates that are known to be present in the vitreous along with others sharing related structures revealed that both goldfish and rat RGCs respond to mannose with an $\mathrm{ED}_{50}$ of $\sim 10 \mu \mathrm{M}$. The responsiveness of goldfish RGCs is stereospecific and requires the positions of the hydroxyl groups on carbon atoms 3, 4, and 5 to be highly constrained; however, the position of the hydroxyl group on carbon 2 is unconstrained, because either mannose or glucose stimulates growth, as does the substitution of an amide group for the hydroxyl group on carbon-2. Goldfish RGCs also respond to a lesser extent when a phosphate is substituted on carbon-6. In the case of rat RGCs, the structural constraints are considerably greater, as indicated by the complete insensitivity of these cells to glucose, glucosamine, or mannose-6-phosphate. Rat RGCs, unlike goldfish RGCs, show a partial response to gulose, perhaps because of its structural similarity to mannose on carbons 2-4. The different response profiles of rat versus goldfish RGCs may represent evolutionary changes in a common receptor, or they could reflect the properties of entirely different receptors in fish and rats. One caveat in these studies is that, because we are using mixed retinal cultures, mannose and/or elevated cAMP could be acting on another cell type first, which releases a secondary factor that acts on RGCs. In goldfish cultures, this seems unlikely, because RGCs constitute up to $70 \%$ of the cells that are present, and their response to AF-1 remains high even at very low plating densities (Schwalb et al., 1995, 1996).

\section{Dissociation of axon-promoting effects and energy metabolism}

Several lines of evidence indicate that the axon-promoting effects of D-mannose or glucose are unrelated to energy metabolism or to cell survival. Our cell cultures already contain high concentrations of galactose and pyruvate, which provide ample carbon sources and which are readily converted to ATP in neurons; the 


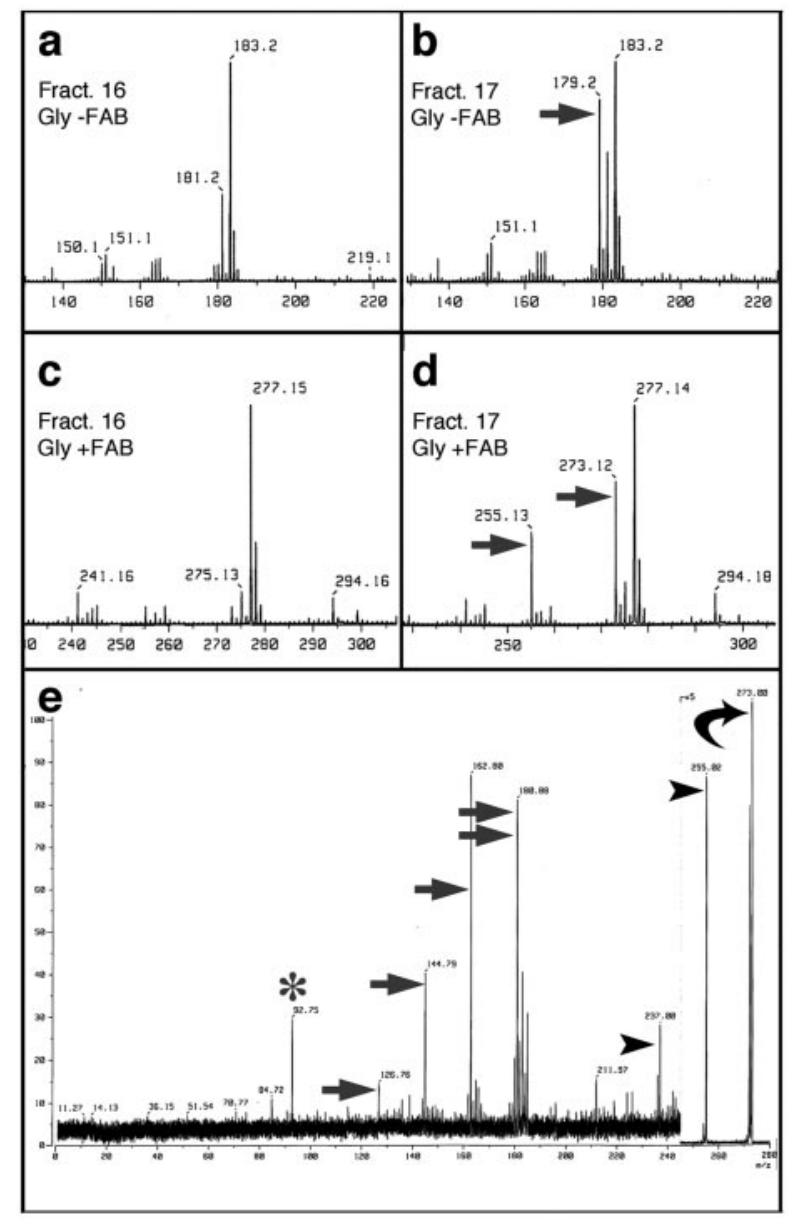

Figure 4. Identification of the axon-promoting factor by mass spectrometry. $a, b$, Fast atomic bombardment mass spectra in the negative ion mode ( $F A B-), m / z$ range of $140-220$, in the presence of glycerol (gly). LC- $\mathrm{NH}_{2}$ column fraction 17, which stimulated outgrowth, contains a peak with $\mathrm{m} / \mathrm{z}=179.2$ ( $b$, arrow) that is not seen in the adjacent, inactive column fraction $(16, a) . \mathrm{m} / \mathrm{z}$ values in the negative ion mode represent the true mass minus 1 proton. $C$, $d$, FAB mass spectra in the positive ion mode ( $F A B+), m / z$ range of $230-300$, in the presence of glycerol. The active fraction $(17, d)$ contains peaks with $\mathrm{m} / \mathrm{z}=273.12$ and 255.13 (arrows) that are absent in the adjacent inactive fraction $(16, c) . \mathrm{m} / \mathrm{z}$ values in the positive ion mode represent the true masses plus one proton. $e, F A B+M S / M S$ analysis of the $m / z=273$ species from $d$ (curved arrow). When subjected to higher voltage, the $\mathrm{m} / \mathrm{z} 273$ species generated a glycerol peak ( $\mathrm{m} / \mathrm{z}=93$; asterisk) and an ion of $\mathrm{m} / \mathrm{z}=181$ (double arrow), i.e., the parent species minus glycerol; most of the additional ions represent successive losses of 18 atomic mass units from either the 181 ion ( $\mathrm{m} / \mathrm{z}=163,145$ and 127; arrows) or from the glycerol adduct of the 181 species ( $\mathrm{m} / \mathrm{z}=255,237$; arrowheads). These results indicate that the axon-promoting factor is a carbohydrate with the formula $\mathrm{C}_{6} \mathrm{H}_{12} \mathrm{O}_{6}$.

low micromolar concentrations of glucose or mannose that cause goldfish RGCs to extend axons are not likely to alter overall ATP levels appreciably and have no effect on cell survival. Another indication that the carbohydrates affect outgrowth per se is the observation that in goldfish RGCs, AF-1 induces the expression of a specific constellation of genes involved in axon growth, including GAP-43, L1, and T $\alpha-1$ tubulin, while not altering the expression of other genes (Petrausch et al., 2000). Moreover, mannoheptulose, an inhibitor of hexose kinases (Rolland et al., 2001), does not block the axon-promoting effects of glucose or mannose, even while depressing cell survival. Thus, the axogenic effect of D-mannose or D-glucose does not require metabolism leading to ATP production, i.e., conversion to the 6- $\mathrm{PO}_{4}$ derivatives, nor does it involve the formation of any further downstream metabolites. The dissociation between axon-promoting effects and energy metabolism is even more apparent in the case of rat RGCs, in which mannose, but not glucose, stimulates outgrowth. The failure of mannoheptulose to block the axonpromoting effects of the carbohydrates also rules out the possibility that the latter could be limiting for glycoprotein synthesis, because formation of the $6-\mathrm{PO}_{4}$ derivatives is also necessary in this regard. Another argument against mannose acting by virtue of its role in glycoprotein synthesis is that glucose is a normal precursor for the carbohydrate side chains in glycoproteins, yet it does not stimulate rat RGCs to regenerate axons even when present at millimolar concentrations. At the same time, the fact that extracellular D-glucose-6- $\mathrm{PO}_{4}$ and D-mannose-6- $\mathrm{PO}_{4}$ can act as weak agonists in goldfish RGCs suggests that they, and by extension mannose and glucose themselves, may act via an extracellular sensor, because the negatively charged phosphate derivatives cannot cross the cell membrane (Abeles et al., 1992). An alternative interpretation of this finding, however, is that either dying cells in culture might release a phosphatase that converts the $6-\mathrm{PO}_{4}$ derivatives to the parent sugars, or that these cells contain an extracellular phosphatase. Whereas most known glucose sensors respond in the low millimolar range (Rolland et al., 2001), the effective concentration here is two orders of magnitude lower. The nature of the carbohydrate sensors responsible for inducing axon outgrowth is as yet unknown.

\section{The role of cAMP}

In addition to differences in carbohydrate responsiveness, goldfish and rat RGCs show intrinsic differences in their requirements for cAMP. The response of goldfish RGCs to mannose or glucose was unaltered by elevating intracellular CAMP concentrations or, up to a point, decreasing the activity of protein kinase A. In contrast, the ability of mature rat RGCs to respond to mannose required elevated intracellular cAMP. This difference is reminiscent of the situation in early developing versus postnatal mammalian peripheral ganglionic neurons: whereas mature mammalian peripheral ganglionic neurons require elevated cAMP to regenerate their axons, immature ganglionic neurons do not (Cai et al., 2001). The exact role of elevated cAMP in our rat RGCs is unknown, but could involve the translocation of a receptor to the cell membrane, as has been described for TrkB in early postnatal rat RGCs (Meyer-Franke et al., 1995; Shen et al., 1999; Goldberg et al., 2002), determining the effects of various extracellular signals on growth cone motility (Ming et al., 1997; Song et al., 1998), and/or modulating a signal transduction pathway or altering gene expression (Cai et al., 2002) such that rat RGCs become competent to respond to mannose.

\section{Role of the glial environment}

Another factor that may contribute to interspecies differences in regenerative potential may be the glial environment (Bastmeyer et al., 1991; Sivron et al., 1994; Wanner et al., 1995). However, altering the intrinsic growth potential of the neuron can partially overcome the negative effects of the glial environment. In one widely studied instance of this phenomenon, sensory ganglion neurons can be stimulated to regenerate their central processes in the mature spinal cord by providing exogenous trophic factors (Ramer et al., 2000), overexpressing GAP-43 and CAP-23 (Bomze et al., 2001), elevating intracellular cAMP (Neumann et al., 2002; Qiu et al., 2002), or transecting the peripheral axons of ganglionic neurons (Neumann and Woolf, 1999). Likewise, although mature rat RGCs are normally unable to regenerate severed axons after optic nerve crush in vivo, they can be stimulated to do so by manipulations that lead to macrophage activation in 


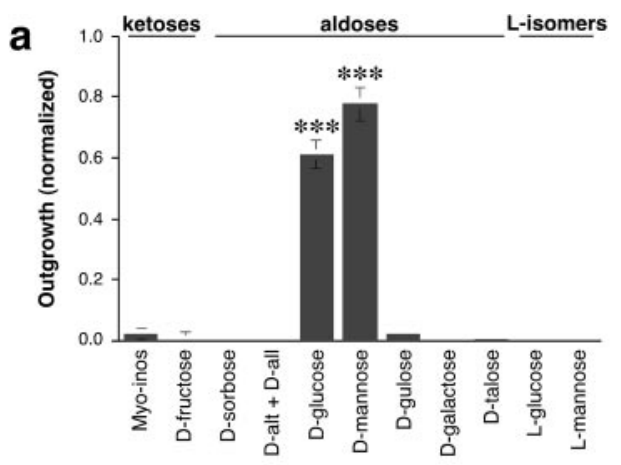

b
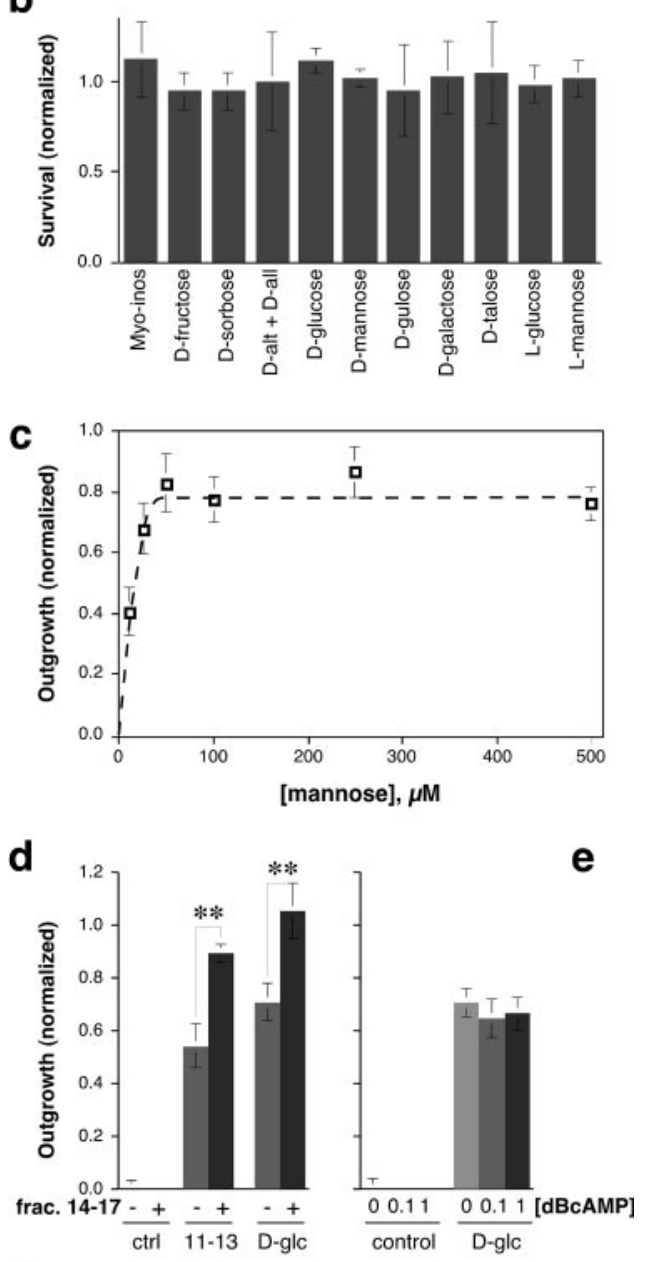

f



Figure 5. Goldfish RGCs respond to specific carbohydrates. a, D-glucose and D-mannose have potent axon-promoting effects. Neither their $\mathrm{L}$-enantiomers nor any other $\mathrm{D}$-sugars are active (tested at 50-100 $\mu \mathrm{m}$ : alt, altrose; all, allose). $b$, None of the samples affects cell survival. The number of viable RGCs per field is normalized to that of untreated controls. $c$, The effect of D-mannose on axon outgrowth saturates at $25-50 \mu \mathrm{m}$, with an $\mathrm{ED}_{50}$ of $\sim 10 \mu \mathrm{M}$. $d$, The maximal effects of the factor isolated from bovine vitreous and of glucose are $\sim 70 \%$ that of unfrac-
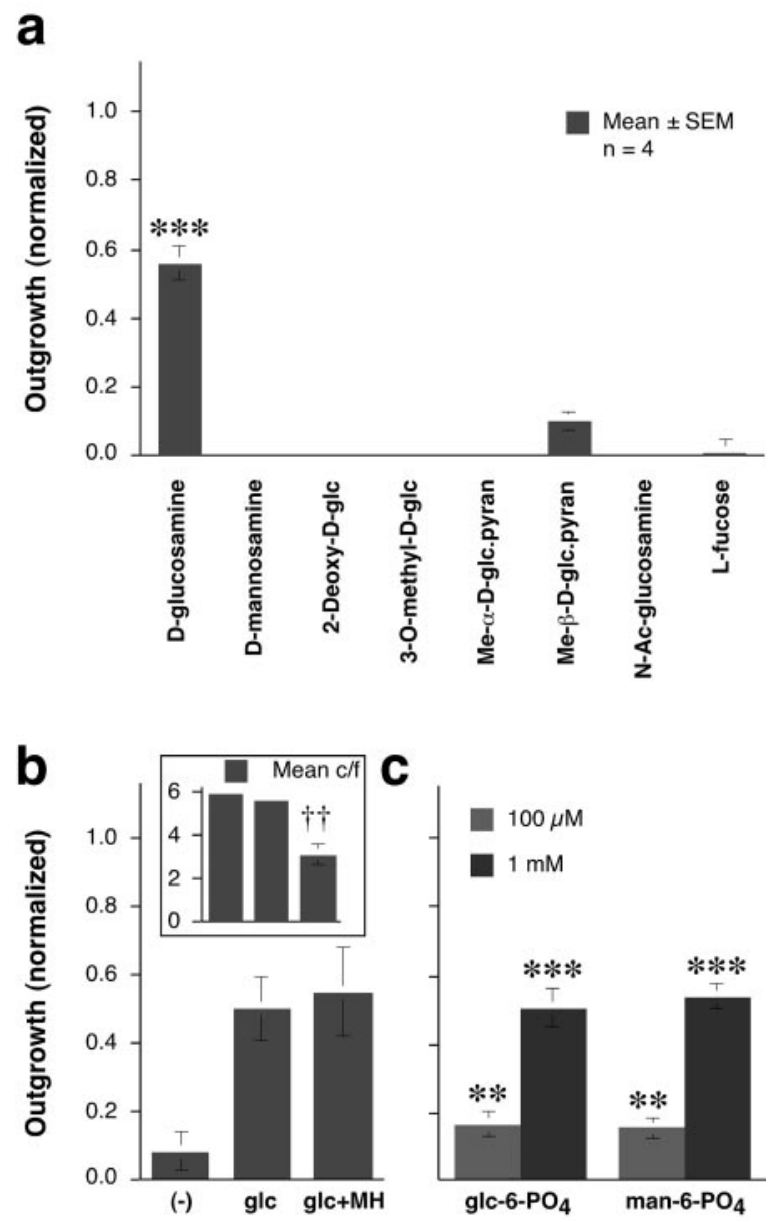

Figure 6. Effects of glucose analogs on goldfish RGCs. $a$, Of the analogs tested, only D-glucosamine ( $25 \mu \mathrm{m}$ ) stimulated axon outgrowth. glc, Glucose; pyran, pyranoside; Ac, acetyl. $b$, Outgrowth is not altered by $\mathrm{MH}$, an inhibitor of glucose-6-kinase and hexose-6-kinase. Inset, MH decreases cell survival (c/f, viable RGCs per field). c, The 6-phosphate derivatives of D-glucose and D-mannose (glc-6-P, man-6-P) stimulate outgrowth when present at 10-fold higher concentrations than the parent hexoses. $y$-axis is the same as in $b .{ }^{* *} p<0.01,{ }^{* * *} p<$ 0.001 relative to cells grown in media alone: ${ }^{t \dagger} p<0.01$ decrease in survival relative to glucose alone.

the eye (Berry et al., 1996; Fischer et al., 2000; Leon et al., 2000; Yin et al., 2003). Under optimal circumstances, RGCs stimulated in this manner can regenerate their axons back to the superior colliculus (Fischer et al., 2001). The present results suggest that such regeneration reflects the contributions of macrophagederived factors, which act in a regulatory capacity, and of mannose that is normally present in the vitreous (Walker and Patrick, 1967), which serves an enabling role.

In summary, our results show that the endogenous low molecular weight factors that stimulate axon outgrowth in goldfish RGCs and that enable mature rat RGCs to grow when other factors are present are carbohydrates. Goldfish RGCs extend axons in response to low micromolar concentrations of glucose, mannose, or glucosamine, even without altering intracellular cAMP levels. The physiological abundance of these sugars may help

tionated $\mathrm{VE}<3$. Another factor that elutes later from the gel-filtration column (14-17), and which has no activity by itself (Fig. $3 d$ ), enhances the effects of glucose and of the carbohydrate isolated from VE <3.e, The membrane-permeable CAMP analog dBCAMP (1 mM) does not augment the effect of glucose. $f$, The protein kinase A inhibitor KT5720 has little effect on mannose-induced growth. 

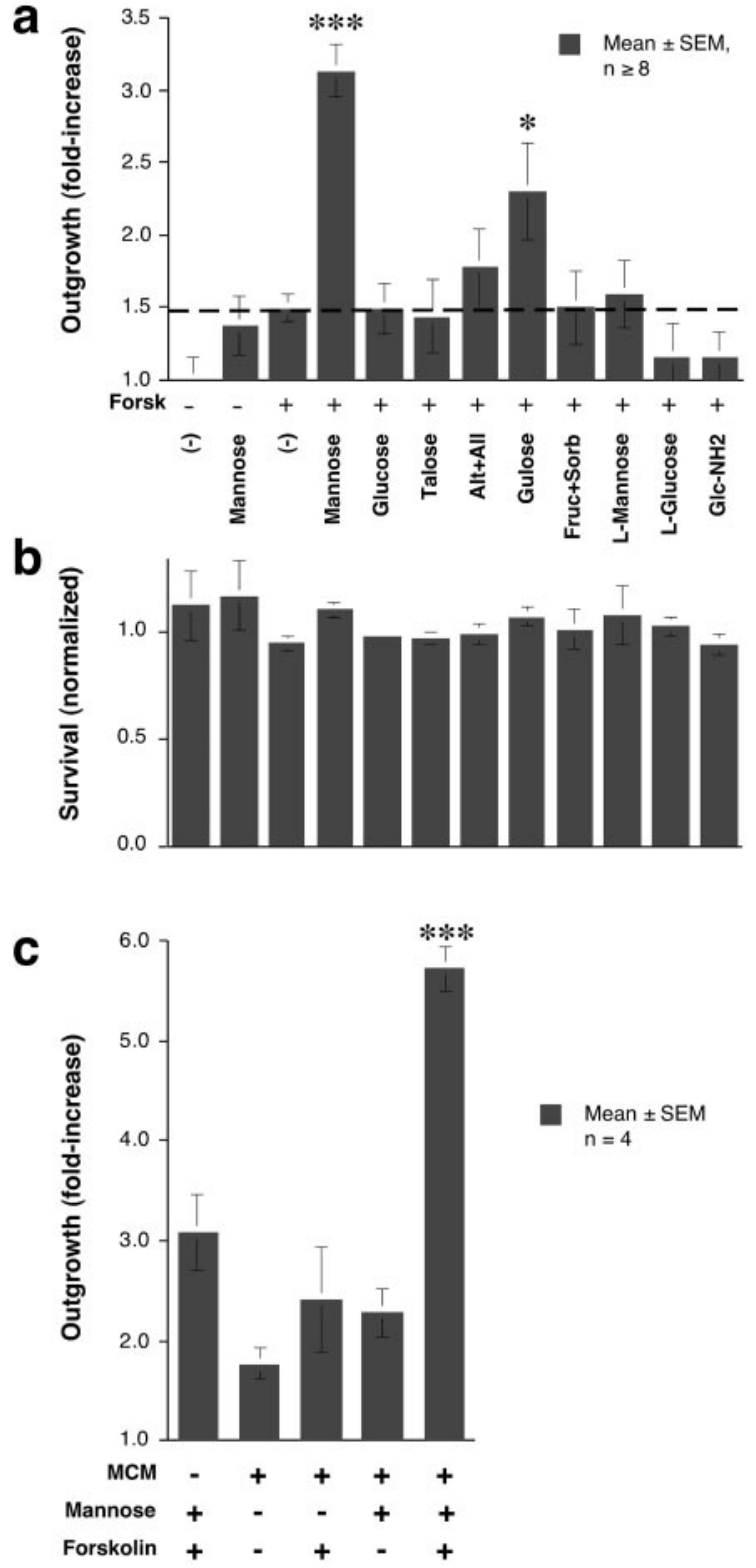

Figure 7. Rat RGCs respond selectively to mannose and require elevated cAMP. $a$, Mature rat $\mathrm{RGCS}$ respond strongly to mannose in the presence of forskolin. Glucose is inactive. All sugars are in the $\mathrm{D}$ - configuration unless noted otherwise. ${ }^{*} p<0.05$, ${ }^{* * *} p<0.001$ compared with forskolin alone (dotted line). $b$, Cell survival is unaffected by any of the carbohydrates. The numbers of viable RGCs per field are normalized to the value in negative controls. c, Proteins $(>$ $3 \mathrm{kDa}$ ) secreted by activated macrophages enhance the effects of mannose. ${ }^{* *} p<0.001$ compared with any of the other conditions.

explain why goldfish can regenerate their optic nerves so readily in vivo. In mammals, although regenerative failure has been ascribed to inhibitory myelin proteins and to the glial scar at the injury site, these barriers can be overcome to a large extent by intravitreal manipulations that cause a macrophage influx into the eye (Yin et al., 2003). The present studies show that micromolar concentrations of mannose are necessary for rat RGCs to respond to macrophage-derived factors and elevated cAMP. Hence, in both lower vertebrates and mammals, specific monosaccharides appear to be essential for axon regeneration.

\section{References}

Abeles RH, Frey PA, Jencks WP (1992) Biochemistry. Boston, MA: Jones and Bartlett.

Aguayo AJ, Rasminsky M, Bray GM, Carbonetto S, McKerracher L, VillegasPerez MP, Vidal-Sanz M, Carter DA (1991) Degenerative and regenerative responses of injured neurons in the central nervous system of adult mammals. Philos Trans R Soc Lond B Biol Sci 331:337-343.

Bastmeyer M, Beckmann M, Schwab ME, Stuermer CA (1991) Growth of regenerating goldfish axons is inhibited by rat oligodendrocytes and CNS myelin but not but not by goldfish optic nerve tract oligodendrocytelike cells and fish CNS myelin. J Neurosci 11:626-640.

Benowitz LI, Jing Y, Tabibiazar R, Jo SA, Petrausch B, Stuermer CA, Rosenberg PA, Irwin N (1998) Axon outgrowth is regulated by an intracellular purine-sensitive mechanism in retinal ganglion cells. J Biol Chem 273:29626-29634.

Berry M, Carlile J, Hunter A (1996) Peripheral nerve explants grafted into the vitreous body of the eye promote the regeneration of retinal ganglion cell axons severed in the optic nerve. J Neurocytol 25:147-170.

Bomze HM, Bulsara KR, Iskandar BJ, Caroni P, Skene JH (2001) Spinal axon regeneration evoked by replacing two growth cone proteins in adult neurons. Nat Neurosci 4:38-43.

Cai D, Qiu J, Cao Z, McAtee M, Bregman BS, Filbin MT (2001) Neuronal cyclic AMP controls the developmental loss in ability of axons to regenerate. J Neurosci 21:4731-4739.

Cai D, Deng K, Mellado W, Lee J, Ratan R, Filbin M (2002) Arginase I and polyamines act downstream from cyclic AMP in overcoming inhibition of axonal growth MAG and myelin in vitro. Neuron 35:711.

Fischer D, Pavlidis M, Thanos S (2000) Cataractogenic lens injury prevents traumatic ganglion cell death and promotes axonal regeneration both in vivo and in culture. Invest Ophthalmol Vis Sci 41:3943-3954.

Fischer D, Heiduschka P, Thanos S (2001) Lens-injury-stimulated axonal regeneration throughout the optic pathway of adult rats. Exp Neurol $172: 257-272$.

Goldberg JL, Espinosa JS, Xu Y, Davidson N, Kovacs GT, Barres BA (2002) Retinal ganglion cells do not extend axons by default: promotion by neurotrophic signaling and electrical activity. Neuron 33:689-702.

Jacobson M (1991) Developmental neurobiology, Ed 3. New York: Plenum.

Leon S, Yin Y, Nguyen J, Irwin N, Benowitz LI (2000) Lens injury stimulates axon regeneration in the mature rat optic nerve. J Neurosci 20:4615-4626.

Meyer-Franke A, Kaplan MR, Pfrieger FW, Barres BA (1995) Characterization of the signaling interactions that promote the survival and growth of developing retinal ganglion cells in culture. Neuron 15:805-819.

Ming GL, Song HJ, Berninger B, Holt CE, Tessier-Lavigne M, Poo MM (1997) cAMP-dependent growth cone guidance by netrin-1. Neuron 19:1225-1235.

Neumann S, WoolfCJ (1999) Regeneration of dorsal column fibers into and beyond the lesion site following adult spinal cord injury. Neuron 23:83-91.

Neumann S, Bradke F, Tessier-Lavigne M, Basbaum AI (2002) Regeneration of sensory axons within the injured spinal cord induced by intraganglionic cAMP elevation. Neuron 34:885-893.

Petrausch B, Tabibiazar R, Roser T, Jing Y, Goldman D, Stuermer CA, Irwin N, Benowitz LI (2000) A purine-sensitive pathway regulates multiple genes involved in axon regeneration in goldfish retinal ganglion cells. J Neurosci 20:8031-8041.

Qiu J, Cai D, Dai H, McAtee M, Hoffman PN, Bregman BS, Filbin MT (2002) Spinal axon regeneration induced by elevation of cyclic AMP. Neuron 34:895-903.

Ramer MS, Priestley JV, McMahon SB (2000) Functional regeneration of sensory axons into the adult spinal cord. Nature 403:312-316.

Ramon y Cajal S (1991) Degeneration and regeneration of the nervous system. New York: Oxford UP.

Rolland F, Winderickx J, Thevelein JM (2001) Glucose-sensing mechanisms in eukaryotic cells. Trends Biochem Sci 26:310-317.

Schwalb JM, Boulis NM, Gu MF, Winickoff J, Jackson PS, Irwin N, Benowitz LI (1995) Two factors secreted by the goldfish optic nerve induce retinal ganglion cells to regenerate axons in culture. J Neurosci 15:5514-5525.

Schwalb JM, Gu MF, Stuermer C, Bastmeyer M, Hu GF, Boulis N, Irwin N, Benowitz LI (1996) Optic nerve glia secrete a low-molecular-weight factor that stimulates retinal ganglion cells to regenerate axons in goldfish. Neuroscience 72:901-910. 
Schwartz M, Agranoff BW (1981) Outgrowth and maintenance of neurites from cultured goldfish retinal ganglion cells. Brain Res 206:331-343.

Shen S, Wiemelt AP, McMorris FA, Barres BA (1999) Retinal ganglion cells lose trophic responsiveness after axotomy. Neuron 23:285-295.

Sivron T, Schwab ME, Schwartz M (1994) Presence of growth inhibitors in fish optic nerve myelin: postinjury changes. J Comp Neurol 343:237-246.

Song H, Ming G, He Z, Lehmann M, McKerracher L, Tessier-Lavigne M, Poo M (1998) Conversion of neuronal growth cone responses from repulsion to attraction by cyclic nucleotides. Science 281:1515-1518.

Tolkovsky AM, Suidan HS (1987) Adenosine 5'-triphosphate synthesis and metabolism localized in neurites of cultured sympathetic neurons. Neuroscience 23:1133-1142.
Volonte C, Rukenstein A, Loeb DM, Greene LA (1989) Differential inhibition of nerve growth factor responses by purine analogues: correlation with inhibition of a nerve growth factor-activated protein kinase. J Cell Biol 109:2395-2403.

Walker F, Patrick RS (1967) Constituent monosaccharides and hexosamine concentration of normal human vitreous humour. Exp Eye Res 6:227-232.

Wanner M, Lang DM, Bandtlow CE, Schwab ME, Bastmeyer M, Stuermer CA (1995) Reevaluation of the growth-permissive substrate properties of goldfish optic nerve myelin and myelin proteins. J Neurosci 15:7500-7508.

Yin Y, Cui Q, Li Y, Irwin N, Fischer D, Harvey AR, Benowitz LI (2003) Macrophage-derived factors stimulate optic nerve regeneration. J Neurosci 23:2284-2293. 